\title{
AN APPROXIMATE ANALYSIS OF OPEN TANDEM QUEUEING NETWORKS WITH BLOCKING AND GENERAL SERVICE TIMES
}

\author{
Kyung P. Jun
}

Center for Communications and Signal Processing Operations Research Program

and

$$
\text { Harry G. Perros }{ }^{1}
$$

Center for Communications and Signal Processing Computer Science Department North Carolina State University Raleigh, NC 27695-8206

CCSP-TR-87/9

June 1987 


\begin{abstract}
An approximation algorithm is presented for open tandem queueing networks with finite buffers and with general service times. The algorithm decomposes the system into individual queues with revised arrival and service process and revised queue capacity. Then, each queue is analyzed in isolation. The service process is revised so that to reflect the additional delay a unit might undergo due to blocking. Unlike previous algorithms, the arrival process to each decomposed queue is described by a $C_{2}$ distribution. The parameters of the service and the arrival processes are computed approximately using an iterative scheme. The approximation procedure yields the steady-state queue-length distribution of each queue. From this, other more commonly sought performance measures, such as mean queue-length, probability a queue is empty, throughput, etc., can be easily computed. Comparisons of the approximate results with simulation results showed that the proposed algorithm has a good error-level.
\end{abstract}




\section{Introduction}

Queueing networks with blocking are in general difficult to treat. In particular, exact closed-form solutions for the steady-state queue-length distributions are not generally attainable except in a few cases. Therefore, analytic approximations, numerical techniques, and simulation techniques are used for the analysis of such queueing networks. Numerical techniques provide the means of analyzing exactly queueing networks. However, when studying queueing networks with blocking, numerical techniques work well for small configurations. For large configurations, time and space complexity problems arise.

The approximation procedures that have been developed so far for open queueing networks with blocking can be classified into the following two categories: 1) The queueing network with blocking is decomposed into individual queues with modified service and arrival process. The service process is revised so as to reflect the additional delay that a customer might undergo due to blocking. The queue capacity is also modified for one particular blocking mechanism. Each queue is then analyzed in isolation. The analysis of each individual queue is carried out using closed-form solutions or numerical solutions depending upon its structure. Several algorithms have been developed using this approach(see for instance Hillier and Boling [15], Altiok and Perros [6], Perros and Snyder [26], Altiok [2, 4, 5], Labetoulle and Pujolle $[19,20]$, Pollock, Birge and Alden [27], Perros and Altiok [25], Takahashi, Miyahara and Hasegawa [28], Kerbache and Smith [16]). These algorithms differ from each other mainly in the way the service process is revised. 
These algorithms have in general minimal cpu time and space requirements. 2) An alternative approach to analyzing queueing networks with blocking is based on the notion of analyzing successive pairs of adjacent queues (see for instance Brandwajn and Jow $[8,9]$, Gershwin $[12,13]$, and Gun [14]). This approach has only been used for tandem configurations. These algorithms differ from each other in the way each pair of adjacent queues is analyzed, and how the results obtained from one pair is used in the analysis of the next pair.

Most of the approximation algorithms in the first group have been proposed for exponential service times. Little work has been done so far on open finite queueing networks with general service times. In particular, Altiok [4] and Pollock, Birge, and Alden [27] considered tandem configurations with general service times, but they assumed that the arrival process to each decomposed queue is a Poisson process. This is not the case. In fact, because of the blocking effect the arrival process at each decomposed queue is not even a renewal process. Labetoulle and Pujolle [19, 20] and Kerbache and Smith [16], analyzed open finite queueing networks with general service times assuming that the arrival process is renewal.

Of the approximation algorithms in the second group, we note that the algorithm due to Gershwin $[12,13]$ is applicable to the case of synchronized machines. All service times are equal to the same constant. Also, machines are subject to breakdown. The algorithm due to Gun [14] allows service times to have a phasetype distribution, but it needs further validation. 
Finally, we note that for closed queueing networks with blocking, Akyildiz and Sieber [1] recently proposed an approximation algorithm for general service times.

In this paper, we present an approximation algorithm for analyzing the performance of open tandem queueing networks with blocking and general service times. This algorithm falls in the first group of algorithms and is an extension of the algorithm due to Altiok [2, 4], Perros and Altiok [25], and Perros and Snyder [26]. However, in these papers the arrival process to each decomposed queue was assumed to be Poisson. In this paper, each arrival process is approximated by a Coxian distribution. The parameters of these distributions are approximated iteratively. We show that this algorithm gives significantly better results than the algorithm reported in Altiok [4]. The algorithm yields the marginal queue-length distributions, from which other performance measures such as mean queue-lengths, server utilization, etc., can be easily obtained.

In the following section, we describe the queueing network under study in detail. Section 3 gives the detailed approximation algorithm. The approximation procedure is validated using simulation in section 4. Finally, the conclusions are given in section 5 .

\section{The Queueing Network Model}

The class of queueing networks with blocking studied here consists of $M$ single server queues in tandem as shown in Figure 2.1. All queues except the first queue 
are assumed to be finite in size. As far as the first queue is concerned, we consider two cases where (i) the first queue has an infinite capacity and (ii) the first queue has a finite capacity. Customers are assumed to arrive only at the first queue in a Poisson fashion with a mean rate $\lambda$. In case (ii) customers arriving during the period that the first queue is full are lost. Customers in each queue are served in FIFO manner. All service times are assumed to be Coxian distributed with two phases (denoted by $C_{2}$ ). That is, a customer in queue $i$ first goes through service phase 1 ; after completing this service, it leaves the service facility with probability $1-\theta_{i}$, while with probability $\theta_{i}$, it receives an additional phase 2 service. Service times in phases 1 and 2 are exponentially distributed with mean $s_{i 1}$ and $s_{i 2}$ respectively. It should be noted that a $C_{2}$ has three independent parameters and squared coefficient of variation $\geq 0.5$. This distribution can be used to approximate general distributions with a rational Laplace transform. In A.1, we describe how the parameters of a $C_{2}$ distribution can be determined using the method of moments. We note that the algorithm presented in this paper can be readily extended to any phase-type service time distributions.

Blocking occurs due to the finite capacity of the queues. When a customer completes its service at the $i$ th queue, it proceeds to the $(i+1)$ st queue if there is a space available. However, if the $(i+1)$ st queue is full at that moment, the customer is forced to wait in front of the $i$ th server until it enters the $(i+1)$ st queue. During this time the $i$ th server remains idle and it cannot serve any other customers that might be waiting in its queue. In this case, the $i$ th queue is said to be blocked, and 


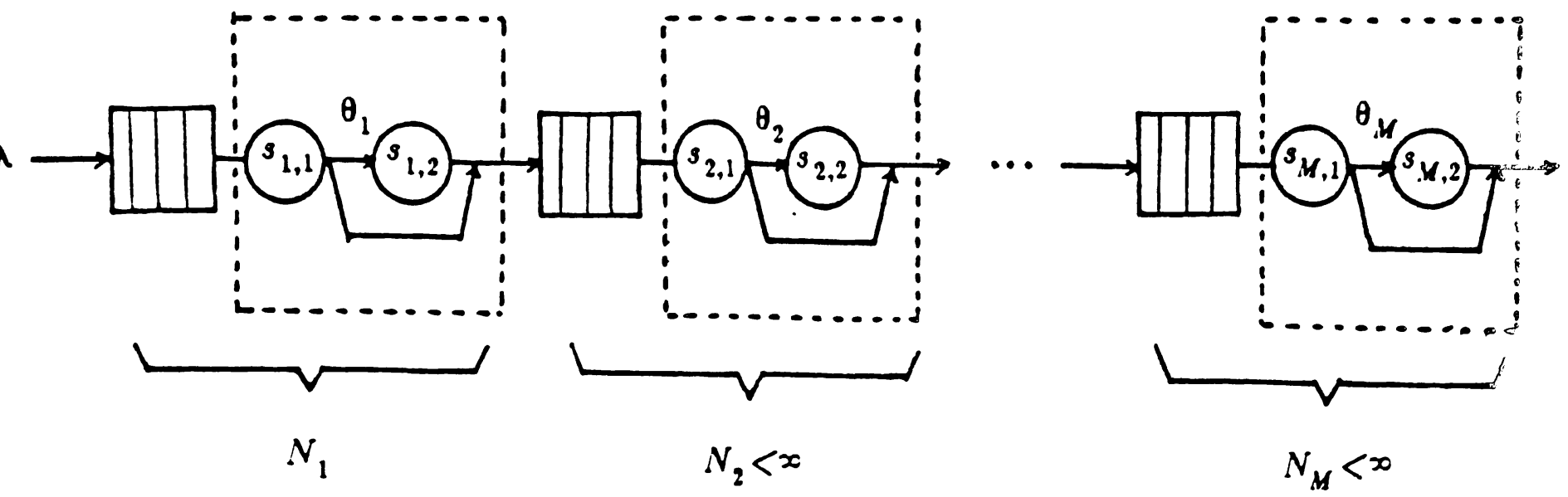

Figure 2.1 The Tandem Configuration

the customer waiting to enter the $(i+1)$ st queue that caused the blocking is referred to as the blocking customer. Obviously, blocking of the $M$ th queue is not feasible in this model. This blocking mechanism is referred to by Onvural and Perros [23] as type 1. One can find a comparison between this blocking mechanism and other mechanisms reported in the literature in Onvural and Perros [23].

Define the following parameters :

$M=$ total number of queues

$\lambda=$ arrival rate to the first queue

$\lambda_{i}=$ overall arrival rate at queue $i$

$N_{i}=$ capacity of queue $i$ (including the one in service). The capacity of the first queue may be finite, equal to $N_{1}$, or infinite.

$s r_{i}=\left(s_{i 1}, s_{i 2}, \theta_{i}\right)$ 
$=$ parameters of the $C_{2}$ service time distribution at queue $i$

$$
\begin{aligned}
a r_{i} & =\left(a_{i 1}, a_{12}, \alpha_{i}\right) \\
& =\text { parameters of the } C_{2} \text { interarrival time distribution at queue } i
\end{aligned}
$$$$
b r_{i}=\left(b_{i 1}, b_{i 2}, \beta_{i}\right)
$$

$=$ parameters of the $C_{2}$ effective service time distribution at queue $i$

$p_{i}(n)=$ probability that there are $n$ units in queue $i$ (including the one in service) at any time

$q_{i}(n)=$ probability that an arriving unit finds $n$ units in queue $i$ (including the one in service)

$r_{i}(n)=$ probability that a departing unit leaves $\mathrm{n}$ units at queue $i$

$w_{i, j}=$ conditional probability that the $i$ th server is at the $j$ th phase of service, given that queue $i$ has $N_{i}$ units, i.e., queue $i$ is full

$\pi_{i}=$ conditional probability that upon service completion at queue $i$ there are $N_{i+1}$ units in queue $(i+1)$, i.e., queue $i+1$ is full

$S_{i}{ }^{*}(s)=$ Laplace transform of the pdf of the original service time at queue $i$

$B_{i}{ }^{*}(s)=$ Laplace transform of the pdf of the effective service time at queue $i$ (including the blocking delay)

\section{The Approximation Algorithm}

In this section, we present the approximation algorithm for the tandem configuration described in Section 2, assuming that the first queue has an infinite queue capacity. We shall refer to this queueing network as Model I. In sub-section 
3.2 , the algorithm is extended to the case where the first queue is finite. We shall refer to this queueing network as Model II.

The approximation algorithm developed in this paper decomposes the system of tandem queues into individual queues with revised queue capacity and revised arrival and service process. These individual queues are then analyzed in isolation. In particular, the capacity of each decomposed queue is augmented by one in order to accommodate the blocking unit. This capacity revision is necessary because the blocking unit, though it physically waits in the blocked queue, is in fact a part of the blocking queue. So the blocking unit is put into the fictitious position at the next buffer and the server at the blocked queue is kept idle until blocking is over.

The interarrival time of units at the first queue is assumed to be Poisson distributed. However, the interarrival time of units at the ith queue $(i \geq 2)$ has an unknown arbitrary distribution because of the blocking effect. The approximation algorithm consists of three basic steps : Step 1, Step 2, and Step 3. In step 1, the arrival process at each finite queue is assumed to be a Poisson process. In steps 2 and 3 , the arrival process is assumed to be a $C_{2}$ distribution. Conceptually, steps 1 and 3 are the same, but implementation for these two steps is different because the arrival process at each queue is different.

\subsection{Model I}

Now, seeing that the first queue is infinite in size, and assuming that it is stable, we have that the departure rate from the first queue is $\lambda$. Thus, the effective 
arrival rate $\bar{\lambda}_{1}$ at queue $i$ is $\bar{\lambda}_{1}=\lambda, i=2,3, \ldots, M$.

\section{Step 1}

This step is practically identical to the algorithm proposed by Perros and Altiok [25], Perros and Snyder [26], and Altiok [2, 4].The non-Poisson arrival process at each decomposed queue $i(i \geq 2)$ is approximated by a Poisson distribution with a parameter $\lambda_{i}$ such that the mean effective arrival rate at this queue is equal to $\lambda$, the system's throughput.

The algorithm begins with the analysis of the Mth queue and proceeds backwards to queue 1 . Because of the network structure, the $M$ th server cannot become blocked. Thus, it can be analyzed in isolation as an $M / C_{2} / 1 / N_{M}+1$ queue.

Now let us consider queue $M-1$. Upon service completion at queue $M-1$, the unit will enter queue $M$ without getting blocked with probability $1-\pi_{M-1}$. Otherwise, with probability $\pi_{M-1}$ it will get blocked. The time spent in blocked state at queue $M-1$ is the remaining service time at queue $M$ at the instance of blocking. When the $(M-1)$ st server gets blocked, the $M$ th server is busy serving a unit and it is either in phase 1 or phase 2 with probability $w_{M, 1}$ and $w_{M, 2}$ respectively. If it is in phase 1 , the distribution of the remaining service time is the service time at queue $M$, i.e., a $C_{2}$ distribution with parameter $\left(s_{M, 1}, s_{M, 2}, \theta_{M}\right)$, because of the memoryless property of the exponential distribution and of the mixture of two exponential distributions. If it is in phase 2 , the remaining service time at phase 2 is distributed exponentially with $1 / s_{M, 2}$. Hence, the effective service time at queue 
$M-1$ consists of two parts : The actual service time at queue $M-1$ and the remaining service time at queue $M$ at the time of blocking (see Figure 3.1).

Now, we can obtain the Laplace transform (LT) of the effective service time distribution at queue $M-1, B_{M-1}(s)$, as follows :

$$
\begin{aligned}
B_{M-1} \dot{\prime}(s)= & \left(1-\pi_{M-1}\right) S_{M-1} \dot{(s)} \\
& +\pi_{M-1}\left[w_{M, 1} B_{M} \dot{*}(s)+w_{M, 2} \frac{s_{M, 2}}{s+s_{M, 2}}\right]
\end{aligned}
$$

where $B_{M}{ }^{*}(s)=S_{M}{ }^{*}(s)$, and

$$
S_{i}^{*}(s)=\left(1-\theta_{i}\right) \frac{s_{i, 1}}{s+s_{i, 1}}+\theta_{i} \frac{s_{i, 1}}{s+s_{i, 1}} \cdot \frac{s_{i, 2}}{s+s_{i, 2}}, i=M-1, M
$$

We can now collapse the distribution of the effective service time into a $C_{2}$ using

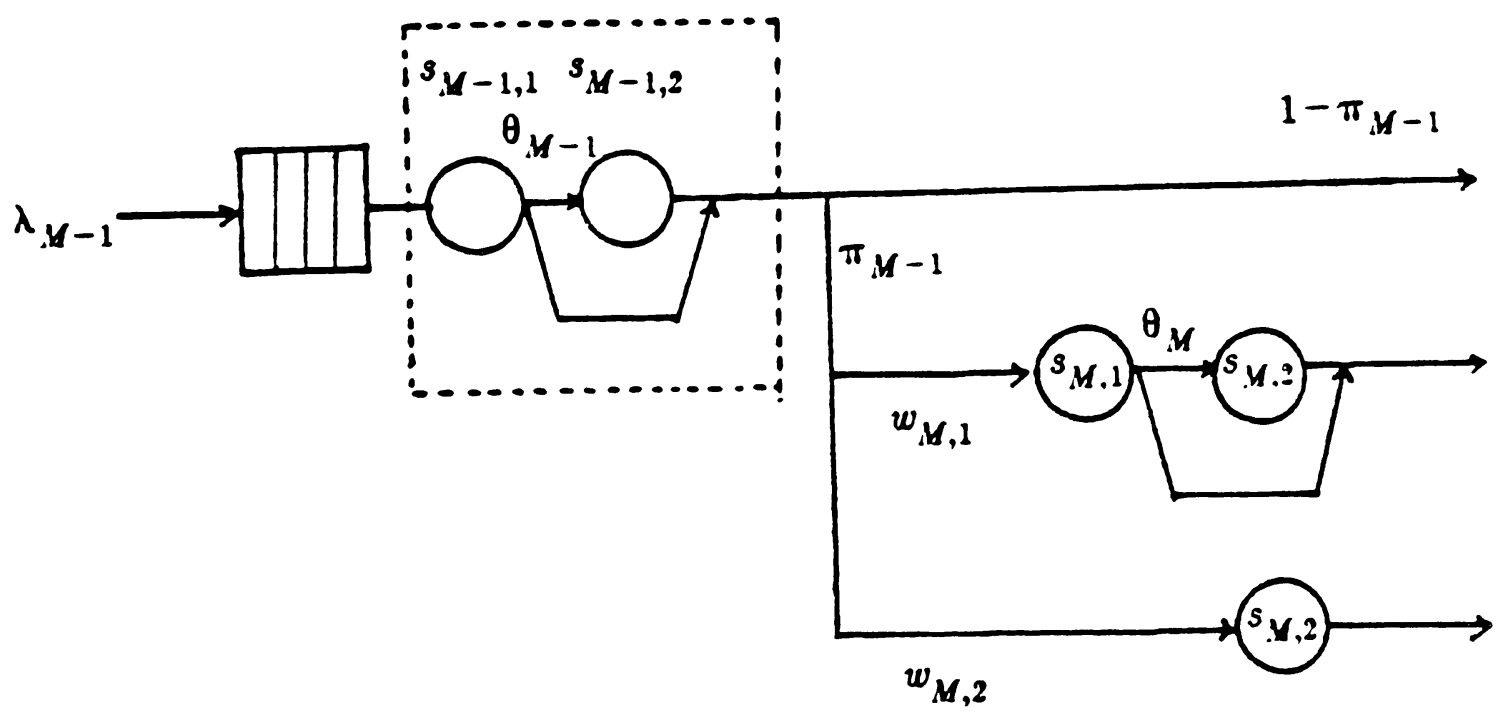

Figure 3.1 The effective service time at queue $M-1$ 
the method of moments (see A.1). This allows us to use only two-phase Coxian distributions, which makes implementation easy and software development feasible. $B_{M-1}(s)$ can be approximated as follows :

$$
B_{M-1}(s) \approx\left(1-\beta_{M-1}\right) \frac{b_{M-1,1}}{s+b_{M-1,1}}+\beta_{M-1} \frac{b_{M-1,1}}{s+b_{M-1,1}} \cdot \frac{b_{M-1,2}}{s+b_{M-1,2}},
$$

where parameters $b_{M-1,1}, b_{M-1,2}$, and $\beta_{M-1}$ can be determined by the threemoment approximation.

The quantities $\pi_{M-1}$ and $w_{M, j}$ are calculated approximately from the queuelength distribution of the $M$ th queue, obtained by analyzing the $M$ th queue in isolation as described above. In particular, the quantity $w_{M, j}$ can be obtained as follows :

$$
w_{M, j}=p_{M}\left(N_{M}, j\right) / p_{M}\left(N_{M}\right)
$$

where $p_{M}\left(N_{M}, j\right)$ is the probability that the $M$ th server is in phase $\mathrm{j}(\mathrm{j}=1,2)$ and there are $N_{M}$ units in queue $M . w_{i, j}$ is, in fact, a probability conditioned on an arrival epoch. However, it is approximated by the arbitrary time probability using the PASTA argument, seeing that the arrival process is assumed to be Poisson. The probability $\pi_{M-1}$ can be obtained using Little's relation on the fictitious position $\left(N_{M}+1\right.$ position $)$ in queue $M$ as follows :

$$
\lambda \pi_{M-1}\left[w_{M, 1}\left(\frac{1}{s_{M, 1}}+\frac{\theta_{M}}{s_{M, 2}}\right)+w_{M, 2} \frac{1}{s_{M, 2}}\right]=p_{M}\left(N_{M}+1\right)
$$

The quantity in the bracket in the above expression is the remaining service time 
of the $M$ th server at the instance the $(M-1)$ st server gets blocked.

We can now analyze queue $M-1$ as an $M / C_{2} / 1 / N_{M-1}+1$ queue using Neuts' matrix-geometric algorithm [22]. In order to do this, we need to know in advance the overall arrival rate $\lambda_{M-1}$ rather than the effective arrival rate $\lambda$. The overall arrival rate $\lambda_{M-1}$, which guarantees a throughput $\lambda$, can be found through the iterative computation of the following fxed-point problem :

$$
\lambda_{M-1}=\lambda /\left[1-p_{M-1}\left(N_{M-1}+1\right)\right] \text {. }
$$

The convergence of the fixed-point problem in (3.5) is guaranteed (see [5]).

Now let us consider queue $M-2$. This queue can be analyzed in the same way as queue $M-1$. In particular, its effective service time can be represented by a $C_{2}$ distribution with parameters $b r_{M-2}=\left(b_{M-2,1}, b_{M-2,2}, \beta_{M-2}\right)$. These parameters can be obtained from the queue-length distribution of the $(M-1)$ st queue, derived approximately as described above. Queue $M-2$ can then be analyzed as an $M / C_{2} / 1 / N_{M-2}+1$ queue. Working backwards in this fashion, we can analyze each queue $i$ in isolation as an $M / C_{2} / 1 / N_{i}+1$ queue. The first queue is analyzed as an $M / C_{2} / 1$. All queues are analyzed using Neuts' matrix-geometric procedure [22].

\section{Step 2}

In step 2, the analysis of the network proceeds from queue 1 to queue $M$. Each queue is analyzed in isolation as in step 1. In particular, the effective service time of each queue is the same as the one calculated in step 1. The capacity of each queue (except queue 1 ) is increased by one as before. The arrival process, unlike 
step 1 , is approximated by a $C_{2}$ distribution. For each decomposed queue, let $d_{i}(t)\left(D_{i}{ }^{*}(s)\right), e_{1}(t)\left(E_{i}{ }^{*}(s)\right)$, and $o_{i}(t)\left(O_{i}{ }^{*}(s)\right)$ be the pdf of the interdeparture time (its LT) from queue $i$, the effective interarrival time (its LT), and the overall interarrival time (its $\mathrm{LT}$ ) at queue $i$, respectively. The arrival process at the first queue is always Poisson distributed. Therefore, in step 2 we do not analyze queue 1.

Now, let us consider queue 2. In order to characterize its arrival process, we must know $d_{1}(t)$, the pdf of the interdeparture time from queue 1 . This is, in general, very difficult to obtain. Instead, we can obtain its LT, $D_{1}{ }^{\prime}(s)$, assuming that successive effective services are independent of each other. When a unit departs from queue 1, either a second unit is available in the queue and ready to be taken into service immediately, or the queue becomes empty. In the first case, the time until the next departure from queue 1 is distributed exactly as the effective service time. On the other hand, if the queue becomes empty, then the time until the next departure is the sum of two intervals, the first being the time until the second unit arrives and the next being his service time. Since these two intervals are independently distributed, the pdf of the sum must be the convolution of the two pdf's. So, we have

$$
D_{1}^{\prime}(s)=\left(1-p_{1}(0)\right) B_{1}^{*}(s)+p_{1}(0) \frac{\lambda}{s+\lambda} \cdot B_{1}^{*}(s)
$$

The above LT of the interdeparture process is in fact equal to $E_{2}{ }^{*}(s)$, the LT of the effective interarrival time at queue 2 . From $E_{2}{ }^{\circ}(s)$, we can obtain the first 
three moments of $e_{2}(t): m_{e 2,1}, m_{e 2,2}$, and $m_{e 2,3}$. In order to analyze queue 2 in isolation we need to know the overall arrival process $o_{2}(t)$ rather than the effective arrival process $e_{2}(t)$. Now, we describe how $o_{2}(t)$ can be obtained approximately from $e_{2}(t)$. Using the decomposition of a renewal process, we have the following relationship between $E_{2}^{\prime \prime}(s)$ and $O_{2}{ }^{\prime}(s)[18]$ :

$$
E_{2}^{\prime}(s)=\frac{\left(1-f_{2}\right) O_{2}^{*}(s)}{1-f_{2} O_{2}^{*}(s)}
$$

where $f_{2}$ is the probability that an arriving unit finds the queue full and is lost, which is equal to $q_{2}\left(N_{2}+1\right)$. This probability can be obtained in terms of the time-average probabilities $p_{2}(n), n=0,1, \ldots, N_{2}+1$ (see A.2). Then, the first three moments of $o_{2}(t), m_{02,1}, m_{02,2}$, and $m_{02,3}$, can be expressed in terms of $f_{2}$ and $m_{e 2,1}, m_{e 2,2}$, and $m_{e 2,3}$ :

$$
\begin{aligned}
& m_{02,1}=\left(1-f_{2}\right) m_{e 2,1}, \\
& m_{02,2}=\left(1-f_{2}\right)\left(m_{e 2,2}-2 f_{2} m_{e 2,1}{ }^{2}\right), \\
& m_{\odot 2,3}=\left(1-f_{2}\right)\left(m_{e 2,3}-6 f_{2} m_{e 2,2} m_{e 2,1}+6 m_{2}^{2} m_{e 2,1}^{3}\right) .
\end{aligned}
$$

Using the above three moments, we can construct a $C_{2}$ representation of $o_{2}(t)$. Queue 2 can now be analyzed as a $C_{2} / C_{2} / 1 / N_{2}+1$ queue with $a r_{2}=\left(a_{2,1}, a_{2,2}, \alpha_{2}\right)$ and $b r_{2}=\left(b_{2,1}, b_{2,2}, \beta_{2}\right)$. We note that the three moments of $o_{2}(t)$ are a function of $f_{2}$, which is unknown. This can be obtained by solving the fixedpoint problem, $1 / m_{02,1}=\lambda /\left(1-f_{2}\right)$. This guarantees that the throughput is equal to $\lambda$. 
For the analysis of queue 3 , we need to know $d_{2}(t)$ or $D_{2}^{\prime}(s)$. $D_{2}^{\prime}(s)$ is obtained in A.3, assuming as before that successive effective service times are independent. We have

$$
D_{2}^{\prime}(s)=\delta_{1} O_{2}^{\prime}(s) B_{2}{ }^{\prime}(s)+\delta_{2} \frac{a_{2,2}}{s+a_{2,2}} B_{2}^{*}(s)+\left(1-\delta_{1}-\delta_{2}\right) B_{2}{ }^{\prime}(s)
$$

where $\delta_{j}$ is the probability that an arrival is in phase $j$ of the arrival process and a departing unit leaves queue 2 empty, $j=1,2 . D_{2}{ }^{\prime}(s)$ is set equal to $E_{3}{ }^{\prime}(s)$. Now, we repeat the same procedure that used for queue 2 to approximate $o_{3}(t)$ by a $C_{2}$ distribution. Now, queue 3 can be analyzed as a $C_{2} / C_{2} / 1 / N_{3}+1$ queue with $a r_{3}=\left(a_{3,1}, a_{3,2}, \alpha_{3}\right)$ and $b r_{3}=\left(b_{3,1}, b_{3,2}, \beta_{3}\right)$.

Following similar arguments the overall arrival process of the $i$ th queue $(2 \leq i \leq M)$ can be represented by a $C_{2}$ distribution. Queue $i$ can then be analyzed as a $C_{2} / C_{2} / 1 / N_{i}+1$ queue with $a r_{i}=\left(a_{i 1}, a_{i 2}, \alpha_{i}\right)$ and $b r_{i}=\left(b_{i 1}, b_{i 2}, \beta_{i}\right)$ in order to obtain the queue-length distribution $p_{i}(n)$. Each queue is step 2 was analyzed numerically using an iterative procedure by Yao and Buzacott [29]. We did not use the matrix-geometric procedure by Neuts [22] as in step 1, because in this case matrix $R$ can only be calculated iteratively. We found that when the traffic intensity is high, the convergence of $R$ is extremely slow.

\section{Step 3}

This step is conceptually identical with step 1 . The objective in this step is to re-calculate the effective service time at each queue. Queue $M$ is not analyzed in 
this step, seeing that it can not be blocked. The analysis, therefore, proceeds from queue $M-1$ to queue 1 . Each queve $i$ is analyzed in isolation as in steps 1 and 2. The capacity of queue $i$ is augmented by one (except queue 1), and the arrival process is taken to be the same as the one calculated is step 2. The effective service time is re-calculated following the same arguments as in step 1. However, due to the fact that we are now dealing with queues with a $C_{2}$ arrival process, rather than a Poisson arrival process, (3.5) expression for $w_{i+1, j}$ needs to be changed as follows:

$$
w_{i+1, j}=q_{i+1}\left(N_{i+1}, j\right) / q_{i+1}\left(N_{i+1}\right)
$$

where $q_{i+1}\left(N_{i+1}, j\right)$ is the probability that the $i+1$ st server is in phase $j(j=1,2)$ and there are $N_{i+1}$ units in queue $i+1$ immediately before an arrival. $q_{i+1}\left(N_{i+1}\right)$ is the probability that there are $N_{i+1}$ units in queue $i+1$ immediately before an arrival. These quantities can be obtained from the steady-state distribution of queue $i$ by conditioning appropriately (see A.2).

As in step 2, we use the iterative procedure by Yao and Buzacott [29] to compute the marginal queue-length distribution $p_{i}(n)$. In order to do this, we need to know the overall arrival process $o_{i}(t)$ rather than the effective arrival process $e_{i}(t)$. Now, we assume that the effective arrival process $e_{i}(t)$ at queue $i$ obtained in step 2 does not change in step 3 . The first three moments of $o_{i}(t)$ can be obtained from (3.8) by appropriately changing the subscripts. We see that the three moments of $o_{i}(t)$ are a function of $f_{i}$, which is the probability that an arriving unit finds the queue full and is lost. This quantity is equal to $q_{i}\left(N_{i}+1\right)$. Because the effective 
service time is recalculated in step 3 , the quantity $f_{1}$ may change. We re-calculate the three moments of $o_{i}(t)$ by solving the fixed-point problem $1 / m_{0 i, 1}=\lambda /\left(1-f_{i}\right)$, where $m_{o i, 1}$ is the Girst moment of $o_{1}(t)$, which guarantees that the throughput is equal to $\lambda$.

The approximation algorithm for Model I can be summarized as follows :

\section{Algorithm for Model I}

\section{Step 1}

0. Set $i=M$.

1. Obtain $B_{i}^{*}(s)$ and $b r_{i}$.

2. Analyze queue $i$ as an $M / C_{2} / 1 / N_{i}+1$ queue and compute $p_{i}{ }^{(1)}(n)$, $n=0,1, \ldots, N_{i}+1, w_{i, j}, j=1,2$, and $\pi_{i-1}$.

3. Set $i=i-1$.

4. If $i=1$, analyze queue 1 as an $M / C_{2} / 1 / \infty$ queue, compute $p_{1}{ }^{(1)}(n), n=0,1, \ldots$, and go to Step 2. Otherwise, go to 1 .

\section{Step 2}

0. Set $i=1$.

1. Obtain $D_{i}^{*}(s)$ and $O_{i+1}(s)$.

2. Calculate the first three moments of $o_{i+1}(t)$ and obtain $a r_{i+1}$.

3. Analyze queue $i$ as a $C_{2} / C_{2} / 1 / N_{i+1}+1$ queue and compute $p_{i+1}{ }^{(2)}(n)$, $n=0,1, \ldots, N_{i+1}+1$.

4. If $i=M-1$, go to Step 3. Otherwise, set $i=i+1$ and go to 1 .

\section{Step 3}

Use the same procedure as in step 1 , but analyze each queue $i,(i \geq 2)$, as a $C_{2} / C_{2} / 1 / N_{i}+1$ queue, and compute $p_{i}{ }^{(3)}(n), n=0,1, \ldots, N_{i}+1$. For the first queue, analyze it as a $M / C_{2} / 1 / \infty$ queue, and compute $p_{1}{ }^{(3)}(n), n=0,1, \ldots$ 


\section{Test for Convergence}

If $\max _{n}\left|p_{i}{ }^{(2)}(n)-p_{i}{ }^{(3)}(n)\right|<\epsilon, i=1,2, \ldots, M$, then stop. Else go back to Step 2.

It should be noted that after the algorithm stops, the actual probability $p_{i}\left(N_{i}\right), i \geq 2$, is obtained by summing $p_{i}\left(N_{i}\right)$ and $p_{i}\left(N_{i}+1\right)$.

\subsection{Model II}

Let us now assume that the first queue is finite. In order to use the previous approximation algorithm, we have to know in advance the effective arrival rate $\bar{\lambda}_{i}$ into each decomposed queue. In the case of Model I, this quantity was readily known as it was equal to the arrival rate $\lambda$ at the first queue. However, in the case of Model II, the effective arrival rate of the first queue is not known. Therefore, the effective arrival rate of the other queues is not readily available. The algorithm presented here is an iterative procedure whereby the true value of the effective arrival rate at the first queue is approximated by successive iterations. Let $\bar{\lambda}$ be the unknown effective arrival rate at the first queue. The iterative procedure first starts with an initial estimate of the value of $\bar{\lambda}$. Following arguments similar to those mentioned in Section 3.1, the queue-length distribution of each decomposed queue is calculated. Using the results for the queue-length distribution of the first queue, a second value for $\bar{\lambda}$ is computed. This value is then used to compute new values for queue-length distribution of each decomposed queue, from which we obtain a third value for $\bar{\lambda}$. The procedure iterates in this fashion until no change in 
the value of $\bar{\lambda}$ is observed Below we give a summary of the iterative procedure for the analysis of Model II.

\section{Algorithm for Model II}

Step 0 (Initialization)

Select a starting value of $\bar{\lambda}$, i.e., $\bar{\lambda}^{(0)}$.

\section{Step 1}

Same as in step 1 for Model I. After queue 1 is analyzed calcylate a new value of $\bar{\lambda}$, i.e., $\lambda^{(1)}$. If $\left|\bar{\lambda}^{(0)}-\bar{\lambda}^{-(1)}\right|<\epsilon$, stop. Otherwise, set $\bar{\lambda}^{-(0)}=\bar{\lambda}^{-(1)}$ and go to Step 2.

\section{Step 2}

Same as in step 2 for Model I, assuming that the effective arrival rate at queue 1 is $\bar{\lambda}^{(0)}$.

\section{Step 3}

Same as in step 3 for Model I, assuming $\bar{\lambda}^{(0)}$ After the first queue is analyzed calculate a new value of $\bar{\lambda}$, i.e., $\bar{\lambda}^{(1)}$. If $\left|\bar{\lambda}^{-(0)}-\bar{\lambda}^{-(1)}\right|<\epsilon$, stop. Otherwise, set $\bar{\lambda}^{(0)}=\bar{\lambda}^{-(1)}$ and go to Step 2.

\section{Numerical Examples}

The approximation algorithm discussed in Section 3 was implemented on a VAX $11 / 785$. It was used to obtain the approximate steady-state queue-length distributions of each queue for three-node and five-node tandem configurations. The approximate results were compared with simulation results obtained using QNAP2 [30]. 
The numerical examples are summarized in Tables 1 to 14. Tables 1 to 4 and 5 to 7 give results respectively for three-node and Gve-node networks with an infinite first queue. Tables 8 to 11 and 12 to 14 give results respectively for threenode and five-node networks with a finite first queue. For presentation purposes, the service distribution at queue $i$ is described in terms of the service rate, $\mu_{i}=1 /\left(1 / s_{i, 1}+\theta_{i} / s_{i, 2}\right)$, and its squared coefficient of variation, $c_{i}{ }^{2}$. The actual distribution used in the experiments is the resulting $C_{2}$ distribution using the approach by Marie [21]. Each table gives the approximate results (column labelled JP), the simulation results, and the results from step 1. This step is identical to the algorithm suggested by Altiok [4] for tandem configurations with general service times. The observed relative error for step 1 and for the whole approximation algorithm are given. Finally, the cpu time used by the approximation algorithm is also given. Due to space considerations, we only give for each queue $i$ the probabilities $p_{i}(0), p_{i}\left(N_{i}\right)$, and mean queue-length, $L_{i}$.

The proposed algorithm gives results which are significantly better than those obtained from step 1. i.e., under the assumption that the arrival process to each decomposed queue is Poisson. In particular, based on the results given in tables 1 to 14 , the average relative error of the approximation algorithm is about $5 \%$, while the average relative error of step 1 is about $13 \%$.

The approximation gives better results when the first queue of the tandem configuration is finite. In particular, based on the results in tables 1 to 14 , the average relative error of the approximation algorithm when the first queue is finite is 
$3 \%$, whereas the average relative error for configurations when the first queue is infinite is $7.5 \%$.

Finally, we note that the algorithm was also used to analyze tandem configurations with exponential service times. No significant improvement over the results obtained using step 1 was observed, when all the service rates were approximately equal. For these balanced cases, the $c^{2}$ of the output process from each queue was close to 1 , thus justifying the Poisson assumption. Some improvement was observed, however, for unbalanced cases.

\section{Conclusions}

An approximation algorithm is presented for open tandem queueing networks with finite buffers and with general service times. The algorithm described in this paper decomposed the system into individual queues with revised arrival and service process and revised queue capacity. The service process is revised so that to reflect the additional delay a unit might undergo due to blocking from the downstream queues. The arrival process is described by a $C_{2}$ distribution. The parameters of the arrival and service processes are computed approximately using an iterative scheme. The algorithm gives results which are significantly better than the results obtained by earlier algorithms in which it was assumed that the arrival process at each decomposed queue was Poisson. Our algorithm performs better when the first queue has a finite capacity when the first queue has an infinite capacity. 
Finally, we note that the approximation algorithm was developed for the case where all service times are $C_{2}$ distributions. The algorithm can be easily extended the case where all service times follow phase-type distributions.

\section{APPENDICES}

\section{A.1. Coxian-2 Distribution}

Consider a Coxian distribution with two phases, denoted by $C_{2}$, and the following parameters $\left(\mu_{1}, \mu_{2}, \theta\right)$. Its Laplace transform is as follows :

$$
f^{\prime}(s)=(1-\theta) \frac{\mu_{1}}{s+\mu_{1}}+\theta \frac{\mu_{1}}{s+\mu_{1}} \cdot \frac{\mu_{2}}{s+\mu_{2}}
$$

Letting $\theta=0$ (or $\theta=1$ ) and $\mu_{1}=\mu_{2}$, we recover the exponential (or the two-stage Erlang) distribution.

The first three moments of a $C_{2}, m_{1}, m_{2}$, and $m_{3}$, can be derived by taking derivatives of $(A-1)$. We have

$$
\begin{aligned}
& m_{1}=\frac{1}{\mu_{1}}+\frac{\theta}{\mu_{2}} \\
& m_{2}=2\left(\frac{1}{\mu_{1}{ }^{2}}+\frac{\theta}{\mu_{1} \mu_{2}}+\frac{\theta}{\mu_{2}{ }^{2}}\right) \\
& m_{3}=6\left(\frac{1}{\mu_{1}{ }^{3}}+\frac{\theta}{\mu_{1}{ }^{2} \mu_{2}}+\frac{\theta}{\mu_{1} \mu_{2}{ }^{2}}+\frac{\theta}{\mu_{2}{ }^{3}}\right)
\end{aligned}
$$

For a general distribution with a squared coefficient of variation $c^{2}>1.0$, given its three moments $m_{1}, m_{2}$, and $m_{3}$, the $C_{2}$ parameters can be determined 
through solving the equations $(A-2)$. Let $\mu_{1}+\mu_{2}=u$ and $\mu_{1} \mu_{2}=v$. With some elementary algebraic manipulation, we have (see [3|)

$$
\begin{aligned}
& u=\left(6 m_{1} m_{2}-2 m_{3}\right) /\left(3 m_{2}{ }^{2}-2 m_{1} m_{3}\right), \\
& v=\left(12 m_{1}{ }^{2}-6 m_{2}\right) /\left(3 m_{2}{ }^{2}-2 m_{1} m_{3}\right), \\
& \mu_{1}, \mu_{2}=\frac{1}{2}\left[u \pm\left(u^{2}-4 v\right)^{1 / 2}\right], \\
& \theta=\mu_{2}\left(m_{1}-1 / \mu_{1}\right),
\end{aligned}
$$

provided that $\frac{m_{3}}{m_{1}{ }^{3}}>1.5\left(c^{2}+1\right)^{2}$ is satisfied. Otherwise, we have infinite number of ways to select the $C_{2}$ parameters. The following set is suggested by Marie [21]:

$$
\mu_{1}=2 / m_{1}, \quad \mu_{2}=1 /\left(m_{1} \cdot c^{2}\right), \quad \theta=1 /\left(2 c^{2}\right)
$$

When the $c^{2}$ is between 0.5 and 1.0, the $C_{2}$ parameters can be determined as follows :

$$
\mu_{1}=1 /\left(m_{1} \cdot c^{2}\right), \quad \mu_{2}=2 / m_{1}, \quad \theta=2\left(1-c^{2}\right)
$$

\section{A.2. Arrival-Epoch Probabilities of a $C_{2} / C_{2} / 1 / N$ queue}

Consider a $C_{2} / C_{2} / 1 / N$ queueing system with parameters ar $=\left(a_{1}, a_{2}, \alpha\right)$ and $b_{r}=\left(b_{1}, b_{2}, \beta\right)$. Let $(n, i, j)$ denote the state of the system, where $n$ is the number of units in the system (including the one in service), $i(i=1,2)$ is the phase number of the arrival process, and $j(j=1,2)$ is the phase number of the service process.

We can now obtain the probability $q(n)$ that an arriving unit finds $n$ units $(n=0,1, \ldots, N)$ in the system, and the probability $q(n, j)$ that the server is in phase $j(j=1,2)$ and there are $\mathrm{n}$ units in the system immediately before an arrival as 
follow :

$$
\begin{aligned}
& q(n, 1)=q(n, 1,1)+q(n, 2,1), \\
& q(n, 2)=q(n, 1,2)+q(n, 2,2), \\
& q(n)=q(n, 1)+q(n, 2),
\end{aligned}
$$

where $q(n, i, j), i=1,2, j=1,2$, is the probability that the system is in state $(n, i, j)$ immediately before an arrival. The probabilities $q(n, i, j), i=1,2, j=1,2$, can be expressed in terms of the time-average probabilities $p(n, i, j)$ as follows :

$$
\begin{aligned}
& q(n, 1, j)=(1-\alpha) a_{1} p(n .1 . j) / H, \quad j=1,2, \\
& q(n, 2, j)=\quad a_{2} p(n, 2, j) / H, \quad j=1,2,
\end{aligned}
$$

where

$$
H=(1-\alpha) a_{1} \sum_{n=0 j=1}^{N} \sum_{n}^{2} p(n, 1, j)+a_{2} \sum_{n=0 j=1}^{N} \sum_{n}^{2} p(n, 2, j)
$$

\section{A.3. Departure Process of a $C_{2} / C_{2} / 1 / N$ Queue}

Consider a $C_{2} / C_{2} / 1 / N$ queueing system with parameters or $=\left(a_{1}, a_{2}, \alpha\right)$ and $b r=\left(b_{1}, b_{2}, \beta\right)$. As in A.2, let $(n, i, j)$ denote a state of the system, where $n(n \geq 0)$ is the number of units in the system, $i(i=1,2)$ is the arrival phase number, and $j(j=1,2)$ is the service phase number. We consider the following cases. 


\begin{tabular}{|c|c|}
\hline $\begin{array}{c}\text { State immediately } \\
\text { after a departure }\end{array}$ & $\begin{array}{c}\text { LT of inter- } \\
\text { departure time }\end{array}$ \\
\hline$(0,1,0)$ & $O^{\prime}(s) B(s)$ \\
\hline$(0,2,0)$ & $\frac{a_{2}}{s+a_{3}} B^{\circ}(s)$ \\
\hline$(n, 1,1), n \geq 1$ & $B^{\prime}(s)$ \\
\hline$(n, 2,1), n \geq 1$ & $B^{\prime}(s)$ \\
\hline
\end{tabular}

Note that $(0, i, 0)$ denotes the state of no unit being in the service facility.

We can now obtain $D^{*}(s)$, the LT of the pdf of the interdeparture time, as follows :

$$
\begin{aligned}
D^{*}(s)= & r(0,1,0) O^{*}(s) B^{*}(s)+r(0,2,0) \frac{a_{2}}{s+a_{2}} B^{*}(s) \\
& +(1-r(0,1,0)-r(0,2,0)) B^{*}(s),
\end{aligned}
$$

where $r(n, i, j)$ is the probability that the system is in state $(n, i, j)$ immediately after a departure. The probabilities $r(0, k, 0), k=1,2$ can be expressed as follows in terms of the time average probabilities $p(n, i, j)$ :

$$
\begin{aligned}
& r(0,1,0)=\frac{1}{G}\left[(1-\beta) b_{1} p(1,1,1)+b_{2} p(1,1,2)\right], \\
& r(0,2,0)=\frac{1}{G}\left[(1-\beta) b_{1} p(1,2,1)+b_{2} p(1,2,2)\right],
\end{aligned}
$$

where

$$
G=(1-\beta) b_{1} \sum_{n=11=1}^{N} \sum_{i}^{2} p(n, i, 1)+b_{2} \sum_{n=1 i=1}^{N} \sum_{i=1}^{2} p(n, i, 2) .
$$


Table 1. $\lambda=2.0, N=(x, 3,3), \mu=(4,4,4), c^{2}=(2,4,8) ; c p u=0.6 \mathrm{sec}$

\begin{tabular}{|lcrrrr|}
\hline & Step 1 & rel err & JP & rel err & simulation \\
\hline$p_{1}(0)$ & 0.249 & 0.361 & 0.168 & -0.082 & 0.183 \\
$L_{1}$ & 5.801 & -0.464 & 9.701 & -0.103 & $10.82(1.456)$ \\
& & & & & \\
$p_{2}(0)$ & 0.333 & 0.085 & 0.297 & -0.033 & 0.307 \\
$p_{2}\left(N_{2}\right)$ & 0.353 & -0.183 & 0.453 & 0.049 & 0.432 \\
$L_{2}$ & 1.500 & -0.104 & 1.731 & 0.034 & $1.674(0.041)$ \\
& & & & & \\
$p_{3}(0)$ & 0.500 & -0.002 & 0.500 & -0.002 & 0.501 \\
$p_{3}\left(N_{3}\right)$ & 0.225 & -0.138 & 0.276 & -0.057 & 0.261 \\
$L_{3}$ & 1.039 & -0.074 & 1.145 & 0.020 & $1.122(0.028)$ \\
\hline
\end{tabular}

Table 2. $\lambda=2.5, N=(\infty, 5,5), \mu=(4,4,4), c^{2}=(2,2,2) ; c p u=0.7 \mathrm{sec}$

\begin{tabular}{|lrrrrr|}
\hline & Step 1 & rel err & JP & rel err & simulation \\
\hline$p_{1}(0)$ & 0.292 & 0.243 & 0.263 & 0.119 & 0.235 \\
$L_{1}$ & 3.234 & -0.403 & 3.758 & -0.307 & $5.420(0.565)$ \\
& & & & & \\
$p_{2}(0)$ & 0.316 & 0.064 & 0.298 & 0.003 & 0.297 \\
$p_{2}\left(N_{2}\right)$ & 0.154 & -0.330 & 0.199 & -0.135 & 0.230 \\
$L_{2}$ & 1.919 & -0.141 & 2.148 & -0.038 & $2.234(0.052)$ \\
& & & & & \\
$p_{3}(0)$ & 0.375 & -0.005 & 0.375 & -0.005 & 0.377 \\
$p_{3}\left(N_{3}\right)$ & 0.113 & -0.271 & 0.142 & -0.084 & 0.155 \\
$L_{3}$ & 1.622 & -0.092 & 1.759 & -0.015 & $1.786(0.033)$ \\
\hline
\end{tabular}


Table 3. $\lambda=2.0, N=(x, 3,5), \mu=(4,4,4), c^{2}=(4,4,4) ; c p u=0.7 \mathrm{sec}$

\begin{tabular}{|lrrrrr|}
\hline & Step 1 & rel err & JP & rel err & simulation \\
\hline$p_{1}(0)$ & 0.347 & 0.295 & 0.290 & 0.082 & 0.268 \\
$L_{1}$ & 3.529 & -0.435 & 4.600 & -0.263 & $6.244(0.794)$ \\
& & & & & \\
$p_{2}(0)$ & 0.440 & 0.071 & 0.413 & 0.005 & 0.411 \\
$p_{2}\left(N_{2}\right)$ & 0.241 & -0.278 & 0.314 & -0.060 & 0.334 \\
$L_{2}$ & 1.162 & -0.149 & 1.336 & -0.022 & $1.366(0.032)$ \\
& & & & & \\
$p_{3}(0)$ & 0.500 & -0.002 & 0.500 & -0.002 & 0.501 \\
$p_{3}\left(N_{3}\right)$ & 0.096 & -0.304 & 0.133 & -0.036 & 0.138 \\
$L_{3}$ & 1.257 & -0.130 & 1.439 & -0.003 & $1.444(0.035)$ \\
\hline
\end{tabular}

Table 4. $\lambda=1.5, N=(\infty, 3,3), \mu=(4,4,4), c^{2}=(8,8,8) ; c p u=0.5 \mathrm{sec}$

\begin{tabular}{|lcrcrr|}
\hline & Step 1 & rel err & JP & rel err & simulation \\
\hline$p_{1}(0)$ & 0.465 & 0.143 & 0.419 & 0.030 & 0.407 \\
$L_{1}$ & 3.058 & -0.323 & 3.711 & -0.179 & $4.519(0.393)$ \\
& & & & & \\
$p_{2}(0)$ & 0.527 & 0.048 & 0.498 & -0.010 & 0.503 \\
$p_{2}\left(N_{2}\right)$ & 0.219 & -0.242 & 0.276 & -0.047 & 0.289 \\
$L_{2}$ & 0.997 & -0.136 & 1.144 & -0.009 & $1.154(0.031)$ \\
& & & & & \\
$p_{3}(0)$ & 0.625 & -0.002 & 0.625 & -0.002 & 0.626 \\
$p_{3}\left(N_{3}\right)$ & 0.139 & -0.295 & 0.178 & -0.011 & 0.180 \\
$L_{3}$ & 0.721 & -0.101 & 0.805 & 0.004 & $0.802(0.021)$ \\
\hline
\end{tabular}


Table 5. $\lambda=1.5, N=(\infty, 3,3,3,3), \mu=(4,4,4,4,4), e^{2}=(2,2,2,2,2)$;

$$
c p u=0.9 \mathrm{sec}
$$

\begin{tabular}{|lrrrrr|}
\hline & Step 1 & rel err & \multicolumn{1}{c|}{ JP } & rel err & simulation \\
\hline$p_{1}(0)$ & 0.587 & 0.046 & 0.580 & 0.034 & 0.561 \\
$L_{1}$ & 0.845 & -0.151 & 0.870 & -0.126 & $0.995(0.040)$ \\
& & & & & \\
$p_{2}(0)$ & 0.588 & 0.044 & 0.577 & 0.025 & 0.563 \\
$p_{2}\left(N_{2}\right)$ & 0.091 & -0.305 & 0.104 & -0.206 & 0.131 \\
$L_{2}$ & 0.697 & -0.133 & 0.737 & -0.083 & $0.804(0.019)$ \\
& & & & & \\
$p_{3}(0)$ & 0.590 & 0.041 & 0.579 & 0.021 & 0.567 \\
$p_{3}\left(N_{3}\right)$ & 0.090 & -0.302 & 0.109 & -0.155 & 0.129 \\
$L_{3}$ & 0.693 & -0.133 & 0.746 & -0.066 & $0.799(0.017)$ \\
& & & & & \\
$p_{4}(0)$ & 0.596 & 0.026 & 0.588 & 0.012 & 0.581 \\
$p_{4}\left(N_{4}\right)$ & 0.087 & -0.293 & 0.106 & -0.138 & 0.123 \\
$L_{4}$ & 0.677 & -0.120 & 0.729 & -0.052 & $0.769(0.015)$ \\
$p_{5}(0)$ & 0.625 & 0.000 & 0.625 & 0.000 & 0.625 \\
$p_{5}\left(N_{5}\right)$ & 0.073 & -0.240 & 0.087 & -0.094 & 0.096 \\
$L_{5}$ & 0.611 & -0.070 & 0.645 & -0.023 & $0.660(0.012)$ \\
\hline
\end{tabular}


Table 8. $\lambda=1.5, N=(x, 4,3,4.3), \mu=(4,3,4,3,4), c^{2}=(2.5,2,1.5,2,2.5)$

$$
c p u=0.9 \mathrm{sec}
$$

\begin{tabular}{|lrrrrr|}
\hline & Step 1 & rel err & \multicolumn{1}{c|}{ JP } & rel err & simulation \\
\hline$p_{1}(0)$ & 0.577 & 0.097 & 0.563 & 0.070 & 0.526 \\
$L_{1}$ & 0.961 & -0.303 & 1.020 & -0.260 & $1.379(0.087)$ \\
& & & & & \\
$p_{2}(0)$ & 0.465 & 0.086 & 0.450 & 0.051 & 0.428 \\
$p_{2}\left(N_{2}\right)$ & 0.102 & -0.396 & 0.126 & -0.254 & 0.169 \\
$L_{2}$ & 1.133 & -0.178 & 1.241 & -0.099 & $1.378(0.029)$ \\
& & & & & \\
$p_{3}(0)$ & 0.575 & 0.055 & 0.563 & 0.033 & 0.545 \\
$p_{3}\left(N_{3}\right)$ & 0.089 & -0.382 & 0.116 & -0.194 & 0.144 \\
$L_{3}$ & 0.709 & -0.173 & 0.782 & -0.088 & $0.857(0.017)$ \\
& & & & & \\
$p_{4}(0)$ & 0.463 & 0.029 & 0.454 & 0.009 & 0.450 \\
$p_{4}\left(N_{4}\right)$ & 0.104 & -0.293 & 0.125 & -0.150 & 0.147 \\
$L_{4}$ & 1.143 & -0.114 & 1.228 & -0.048 & $1.290(0.026)$ \\
& & & & & \\
$p_{5}(0)$ & 0.625 & -0.003 & 0.625 & -0.003 & 0.627 \\
$p_{5}\left(N_{5}\right)$ & 0.083 & -0.217 & 0.098 & -0.075 & 0.106 \\
$L_{5}$ & 0.630 & -0.065 & 0.665 & 0.013 & $0.674(0.013)$ \\
\hline
\end{tabular}


Table 7. $\lambda=1.2, N=(\infty, 5,5,5,5), \mu=(2,2,2,2,2), c^{2}=(1.5,1.5,1.5,1.5,1.5)$

$$
c p u=0.8 \mathrm{sec}
$$

\begin{tabular}{|lrrrrr|}
\hline & Step 1 & rel err & \multicolumn{1}{c}{ JP } & rel err & simulation \\
\hline$p_{1}(0)$ & 0.351 & 0.170 & 0.339 & 0.130 & 0.300 \\
$L_{1}$ & 2.140 & -0.326 & 2.265 & -0.286 & $3.174(0.240)$ \\
& & & & & \\
$p_{2}(0)$ & 0.352 & 0.128 & 0.339 & 0.087 & 0.312 \\
$p_{2}\left(V_{2}\right)$ & 0.105 & -0.420 & 0.127 & -0.298 & 0.181 \\
$L_{2}$ & 1.651 & -0.186 & 1.783 & -0.121 & $2.029(0.053)$ \\
& & & & & \\
$p_{3}(0)$ & 0.355 & 0.096 & 0.344 & 0.062 & 0.324 \\
$p_{3}\left(N_{3}\right)$ & 0.103 & -0.387 & 0.125 & -0.256 & 0.168 \\
$L_{3}$ & 1.635 & -0.165 & 1.768 & -0.097 & $1.958(0.045)$ \\
& & & & & \\
$p_{4}(0)$ & 0.365 & 0.046 & 0.357 & 0.023 & 0.349 \\
$p_{4}\left(N_{4}\right)$ & 0.097 & -0.322 & 0.117 & -0.182 & 0.143 \\
$L_{4}$ & 1.588 & -0.122 & 1.705 & -0.057 & $1.808(0.039)$ \\
& & & & & \\
$p_{5}(0)$ & 0.400 & 0.005 & 0.400 & 0.005 & 0.398 \\
$p_{5}\left(N_{5}\right)$ & 0.078 & -0.250 & 0.092 & -0.115 & 0.104 \\
$L_{5}$ & 1.428 & -0.078 & 1.504 & -0.029 & $1.549(0.026)$ \\
\hline
\end{tabular}


Table 8. $\lambda=3.0, N=(3,3,3), \mu=(4,4,4), c^{2}=(2,4,8) ; c p u=0.2 \mathrm{sec}$

\begin{tabular}{|lrrrrr|}
\hline & Step 1 & rel err & \multicolumn{1}{c}{ JP } & rel err & simulation \\
\hline$p_{1}(0)$ & 0.262 & 0.036 & 0.244 & -0.036 & 0.253 \\
$p_{1}\left(N_{1}\right)$ & 0.341 & -0.034 & 0.361 & 0.023 & 0.353 \\
$L_{1}$ & 1.606 & -0.024 & 1.668 & 0.013 & $1.646(0.016)$ \\
& & & & & \\
$p_{2}(0)$ & 0.342 & 0.018 & 0.337 & 0.003 & 0.336 \\
$p_{2}\left(N_{2}\right)$ & 0.344 & -0.080 & 0.386 & 0.032 & 0.374 \\
$L_{2}$ & 1.471 & -0.043 & 1.559 & 0.014 & $1.537(0.018)$ \\
& & & & & \\
$p_{3}(0)$ & 0.506 & -0.019 & 0.521 & 0.010 & 0.516 \\
$p_{3}\left(N_{3}\right)$ & 0.221 & -0.075 & 0.250 & 0.046 & 0.239 \\
$L_{3}$ & 1.024 & -0.028 & 1.069 & 0.015 & $1.053(0.017)$ \\
\hline
\end{tabular}

Table 9. $\lambda=4.0, N=(5,5,5), \mu=(4,4,4), c^{2}=(2,2,2) ; c p u=0.2 \mathrm{sec}$

\begin{tabular}{|lrrrrr|}
\hline & Step 1 & rel err & \multicolumn{1}{c|}{ JP } & rel err & simulation \\
\hline$p_{1}(0)$ & 0.130 & 0.182 & 0.116 & 0.055 & 0.110 \\
$p_{1}\left(N_{1}\right)$ & 0.292 & -0.058 & 0.310 & 0.000 & 0.310 \\
$L_{1}$ & 2.986 & -0.044 & 3.088 & -0.012 & $3.125(0.033)$ \\
& & & & & \\
$p_{2}(0)$ & 0.196 & 0.016 & 0.197 & 0.021 & 0.193 \\
$p_{2}\left(N_{2}\right)$ & 0.278 & -0.145 & 0.319 & -0.018 & 0.325 \\
$L_{2}$ & 2.654 & -0.060 & 2.798 & -0.009 & $2.822(0.037)$ \\
& & & & & \\
$p_{3}(0)$ & 0.292 & -0.070 & 0.310 & -0.013 & 0.314 \\
$p_{3}\left(N_{3}\right)$ & 0.175 & -0.129 & 0.198 & -0.015 & 0.201 \\
$L_{3}$ & 2.051 & -0.027 & 2.115 & 0.003 & $2.108(0.034)$ \\
\hline
\end{tabular}


Table 10. $\lambda=4.0, N=(5,3,5), \mu=(3,5,3), c^{2}=(4,4,4) ; c p u=0.4 \mathrm{sec}$

\begin{tabular}{|lrrrrr|}
\hline & Step 1 & rel err & JP & rel err & simulation \\
\hline$p_{1}(0)$ & 0.084 & 0.424 & 0.066 & 0.119 & 0.059 \\
$p_{1}\left(N_{1}\right)$ & 0.463 & -0.065 & 0.495 & 0.000 & 0.495 \\
$L_{1}$ & 3.553 & -0.056 & 3.711 & -0.014 & $3.763(0.026)$ \\
& & & & & \\
$p_{2}(0)$ & 0.395 & 0.045 & 0.386 & 0.021 & 0.378 \\
$p_{2}\left(N_{2}\right)$ & 0.293 & -0.219 & 0.367 & -0.021 & 0.375 \\
$L_{2}$ & 1.313 & -0.116 & 1.464 & -0.015 & $1.486(0.028)$ \\
& & & & & \\
$p_{3}(0)$ & 0.283 & -0.135 & 0.326 & -0.003 & 0.327 \\
$p_{3}\left(V_{3}\right)$ & 0.252 & -0.154 & 0.296 & -0.007 & 0.298 \\
$L_{3}$ & 2.287 & -0.036 & 2.388 & -0.006 & $2.373(0.049)$ \\
\hline
\end{tabular}

Table 11. $\lambda=4.0, N=(3,3,3), \mu=(4,4,4), c^{2}=(8,8,8) ; c p u=0.2 \mathrm{sec}$

\begin{tabular}{|lrrrrr|}
\hline & Step 1 & rel err & JP & rel err & simulation \\
\hline$p_{1}(0)$ & 0.209 & 0.118 & 0.172 & -0.080 & 0.187 \\
$p_{1}\left(N_{1}\right)$ & 0.507 & -0.032 & 0.540 & 0.031 & 0.524 \\
$L_{1}$ & 1.936 & -0.034 & 2.051 & 0.023 & $2.004(0.019)$ \\
& & & & & \\
$p_{2}(0)$ & 0.344 & 0.033 & 0.340 & 0.021 & 0.333 \\
$p_{2}\left(N_{2}\right)$ & 0.382 & -0.128 & 0.469 & 0.071 & 0.438 \\
$L_{2}$ & 1.527 & -0.073 & 1.692 & 0.027 & $1.647(0.034)$ \\
& & & & & \\
$p_{3}(0)$ & 0.507 & -0.047 & 0.540 & 0.015 & 0.532 \\
$p_{3}\left(N_{3}\right)$ & 0.220 & -0.134 & 0.272 & 0.071 & 0.254 \\
$L_{3}$ & 1.022 & -0.036 & 1.088 & 0.026 & $1.060(0.028)$ \\
\hline
\end{tabular}


Table 12. $\lambda=3.0, N=(3,3,3,3,3), \mu=(4,4,4,4,4), c^{2}=(2,2,2,2,2)$;

$$
c p u=0.6 \mathrm{sec}
$$

\begin{tabular}{|lrrrrr|}
\hline & Step 1 & rel err & JP & rel err & simulation \\
\hline$p_{1}(0)$ & 0.287 & 0.148 & 0.270 & 0.080 & 0.250 \\
$p_{1}\left(V_{1}\right)$ & 0.281 & -0.060 & 0.297 & -0.007 & 0.299 \\
$L_{1}$ & 1.482 & -0.060 & 1.535 & -0.027 & $1.577(0.018)$ \\
& & & & & \\
$p_{9}(0)$ & 0.307 & 0.141 & 0.291 & 0.082 & 0.269 \\
$p_{2}\left(V_{2}\right)$ & 0.317 & -0.174 & 0.355 & -0.076 & 0.384 \\
$L_{2}$ & 1.495 & -0.103 & 1.586 & -0.049 & $1.667(0.024)$ \\
& & & & & \\
$p_{3}(0)$ & 0.334 & 0.077 & 0.326 & 0.052 & 0.310 \\
$p_{3}\left(V_{3}\right)$ & 0.287 & -0.185 & 0.325 & -0.077 & 0.352 \\
$L_{3}$ & 1.404 & -0.095 & 1.484 & -0.043 & $1.551(0.024)$ \\
& & & & & \\
$p_{4}(0)$ & 0.376 & 0.021 & 0.377 & 0.024 & 0.368 \\
$p_{4}\left(N_{4}\right)$ & 0.245 & -0.167 & 0.273 & -0.071 & 0.294 \\
$L_{4}$ & 1.270 & -0.072 & 1.321 & -0.035 & $1.369(0.022)$ \\
$p_{5}(0)$ & 0.461 & -0.023 & 0.473 & 0.002 & 0.472 \\
$p_{5}\left(N_{5}\right)$ & 0.174 & -0.130 & 0.190 & -0.050 & 0.200 \\
$L_{5}$ & 1.023 & -0.033 & 1.041 & -0.016 & $1.058(0.019)$ \\
\hline
\end{tabular}


Table 13. $\lambda=1.5, N=(4,3,4,3,4), \mu=(2.5,2,2.5,2,2.5), c^{2}=(2.5,2,2.5,2,2.5)$;

$$
c p u=0.1 \mathrm{sec}
$$

\begin{tabular}{|lrrrrr|}
\hline & Step 1 & rel err & \multicolumn{1}{c|}{ JP } & rel err & simulation \\
\hline$p_{1}(0)$ & 0.317 & 0.187 & 0.288 & 0.079 & 0.267 \\
$p_{1}\left(N_{1}\right)$ & 0.187 & -0.126 & 0.207 & -0.033 & 0.214 \\
$L_{1}$ & 1.675 & -0.104 & 1.789 & -0.043 & $1.870(0.023)$ \\
& & & & & \\
$p_{2}(0)$ & 0.384 & 0.088 & 0.271 & 0.038 & 0.261 \\
$p_{2}\left(N_{2}\right)$ & 0.345 & -0.177 & 0.396 & -0.055 & 0.419 \\
$L_{2}$ & 1.573 & -0.092 & 1.682 & -0.029 & $1.732(0.022)$ \\
& & & & & \\
$p_{3}(0)$ & 0.361 & 0.097 & 0.344 & 0.046 & 0.329 \\
$p_{3}\left(N_{3}\right)$ & 0.191 & -0.257 & 0.234 & -0.089 & 0.257 \\
$L_{3}$ & 1.567 & -0.133 & 1.723 & -0.047 & $1.808(0.031)$ \\
& & & & & \\
$p_{4}(0)$ & 0.343 & -0.026 & 0.349 & -0.009 & 0.352 \\
$p_{4}\left(N_{4}\right)$ & 0.280 & -0.152 & 0.316 & -0.042 & 0.330 \\
$L_{4}$ & 1.377 & -0.051 & 1.438 & -0.009 & $1.451(0.018)$ \\
& & & & & \\
$p_{5}(0)$ & 0.512 & -0.028 & 0.524 & -0.006 & 0.527 \\
$p_{5}\left(N_{5}\right)$ & 0.093 & -0.155 & 0.107 & -0.027 & 0.110 \\
$L_{5}$ & 1.019 & -0.037 & 1.053 & -0.005 & $1.058(0.018)$ \\
\hline
\end{tabular}


Table 14. $\lambda=3.0, N=(5,5,5,5,5), \mu=(4,4,4,4,4), c^{2}=(1.5,1.5,1.5,1.5,1.5)$;

$$
c p u=0.3 \mathrm{sec}
$$

\begin{tabular}{|lrrrrr|}
\hline & Step 1 & rel err & \multicolumn{1}{c}{ JP } & rel err & simulation \\
\hline$p_{1}(0)$ & 0.259 & 0.088 & 0.248 & 0.042 & 0.238 \\
$p_{1}\left(N_{1}\right)$ & 0.128 & -0.092 & 0.135 & -0.043 & 0.141 \\
$L_{1}$ & 2.049 & -0.059 & 2.107 & -0.032 & $2.177(0.034)$ \\
& & & & & \\
$p_{2}(0)$ & 0.264 & 0.078 & 0.253 & 0.033 & 0.245 \\
$p_{2}\left(N_{2}\right)$ & 0.173 & -0.203 & 0.198 & -0.088 & 0.217 \\
$L_{2}$ & 2.128 & -0.089 & 2.257 & -0.034 & $2.336(0.045)$ \\
& & & & & \\
$p_{3}(0)$ & 0.274 & 0.050 & 0.266 & 0.019 & 0.261 \\
$p_{3}\left(N_{3}\right)$ & 0.163 & -0.231 & 0.190 & -0.104 & 0.212 \\
$L_{3}$ & 2.069 & -0.091 & 2.198 & -0.035 & $2.277(0.047)$ \\
& & & & & \\
$p_{4}(0)$ & 0.294 & 0.000 & 0.291 & -0.010 & 0.294 \\
$p_{4}\left(N_{4}\right)$ & 0.146 & -0.193 & 0.168 & -0.072 & 0.181 \\
$L_{4}$ & 1.953 & -0.065 & 2.056 & -0.015 & $2.088(0.041)$ \\
& & & & & \\
$p_{5}(0)$ & 0.346 & -0.034 & 0.351 & -0.020 & 0.358 \\
$p_{5}\left(N_{5}\right)$ & 0.109 & -0.155 & 0.123 & -0.047 & 0.129 \\
$L_{5}$ & 1.683 & -0.032 & 1.741 & 0.002 & $1.738(0.031)$ \\
\hline
\end{tabular}




\section{REFERENCES}

[1] Akyildiz, I.F. and A. Sieber, "General Closed Queueing Networks with Blocking," Tech. Rep. 87-015, Dep't of CS, LSU (1987)

[2] Altiok, T., "Approximate Analysis of Exponential Tandem Queues with Blocking, " European J. Oper. Res., 11, 390-398 (1982)

[3] Altiok, T., "On the Phase-Type Approximations of General Distributions," AIIE Trans., 17, 110-116 (1985)

[4] Altiok, T., "Approximate Analysis of Queues in Series with Phase-type Service Times and Blocking," IE Rep., Rutgers Univ. (1986)

[5] Altiok, T. and H.G. Perros, "Open Networks of Queues with Blocking: Split and Merge Configurations," IIE Trans., 251-261 (1986)

[6] Altiok, T. and H.G. Perros, "Approximate Analysis of Arbitrary Configurations of Queueing . Networks with Blocking," Annals of OR, 481-509 (1987)

[7] Boxma, O. and A. Konheim, "Approximate Analysis of Exponential Queueing Systems with Blocking," Acta Informatica, 15, 19-66 (1981)

[8] Brandwajn, A. and Y.L. Jow, "An Approximation Method for Tandem Queues with Blocking," Amdhal Corp., Tech. Rep. (1985a)

[9] Brandwajn, A. and Y.L. Jow, "Tandem Exponential Queues with Finite Buffers," Int. Sem. on Comp. Network and Perf. Eval., Tokyo (1985b)

(10) Caseau, P. and G. Pujolle, "Throughput Capacity of a Sequence of Queues with Blocking due to Finite Waiting Room," IEEE Trans. Software Eng., SE5, 631-642 (1979)

[11] Foster, F.G. and H.G. Perros, "On the Blocking Process in Queueing Networks, " European J. Oper. Res., 5, 276-283 (1980)

[12] Gershwin, S.B., "An Efficient Decomposition Method for the Approximate Evaluation of Production Lines with Finite Storage Space," Tech. Rep. LISD-P-1308, MIT (1983)

[13] Gershwin, S.B., "Representation and Analysis of Transfer Lines with Machines that Have Different Processing Rates," Tech. Rep. LIDS-P-1446, MIT (1985)

[14] Gun, L., "Tandem Queueing Systems Subject to Blocking with Phase-type Servers: Analytic Solutions and Approximations," Tech. Rep. TR-87-02, Systems Res. Center, Univ. of Maryland (1987)

[15] Hillier, F.S. and R. Boling, "Finite Queues in Series with Exponential or Erlang Service Times - A Numerical Approach," Oper. Res., 15, 286-303 (1967)

[16] Kerbache, L. and J.M. Smith, "The Generalized Expansion Method for Open Finite Queueing Networks," Tech. Rep., Dep't of IE and OR, Univ. of Mass. 
(1986)

[17] Konheim, A. and .M. Reiser, "A Queueing Model with Finite Waiting Room and Blocking," J. ACM, 23, 328-341 (1976)

[18] Kuehn, P.J., "Approximate Analysis of General . Vetworks by Decomposition," IEEE Trans. Comm., COM-27, 113-126 (1979)

[19] Labetoulle, J. and G. Pujolle, "Modelling of Packet Switching Communication Networks with Finite Buffers Size at Each Node," Computer Performance, Chandy and Reiser(eds.), North-Holland, 515-535 (1977)

[20] Labetoulle, J. and G. Pujolle, "Isolation Method in a Network of Queues," IEEE Tran. Soft. Eng., SE-6, 373-381 (1980)

[21] Marie, R., "Calculating Equilibrium Probabilities for $\lambda(\mathrm{n}) / C_{k} / 1 / \mathrm{N}$ Queues," Performance'80 (1980)

[22] Neuts, M.F., Matrix-Geometric Solutions in Stochastic Models - An Algorithmic Approach, The Johns-Hopkins Univ. Press (1981)

[23] Onvural, R.O. and H.G. Perros, "On Equivalencies of Blocking Mechanisms in Queueing Networks with Blocking," Operations Research Letters, 293-297 (1986)

[24] Perros, H.G., "A Symmetrical Exponential Open Queue Network with Blocking and Feedback, " EEE Trans. Soft. Eng., SE-7, 395-402 (1981)

[25] Perros, H.G. and T. Altiok, "Approximate Analysis of Open Networks of Queues with Blocking : Tandem Configurations," IEEE Trans. Soft. Eng., 450-461 (1986)

[26] Perros, H.G. and P. Snyder, "Computationally Efficient Approximation Algorithm for Analyzing Open Queueing Networks with Blocking," CS Rep., NC State Univ. (1986)

[27] Pollock, S.M., J.R. Birge and J.M. Alden, "Approximate Analysis for Open Tandem Queues with Blocking : Exponential and General Service Time Distribution," Tech. Rep. 85-30, Dep't of Ind. and Oper. Eng., Univ. of Michigan (1985)

[28] Takahashi, Y., H. Miyahara and T. Hasegawa, "An Approximation Method for Open Restricted Queueing Networks, " Oper. Res., 28, 594-602 (1980)

[29] Yao, D.D. and J.A. Buzacott, "Queueing Models for a Flexible Machining Station Part II : The Method of Coxian phases," European J. of Oper. Res., 19, 241-252 (1985)

[30] Veran, M. and D. Potier, "A Portable Environment for Queueing Systems Modelling," INRLA rep. 314 (1984) 